\title{
Diagnostic and Analysis of Human Sperm Characteristics Using Fourier Transform Infrared Spectroscopy
}

\author{
Amir Abramovich, Alexander Shulzinger \\ Department of Electrical and Electronic Engineering, Ariel University, Ariel, Israel \\ Email: amir007@ariel.ac.il
}

Received 23 March 2015; accepted 27 June 2015; published 30 June 2015

Copyright (C) 2015 by authors and Scientific Research Publishing Inc.

This work is licensed under the Creative Commons Attribution International License (CC BY).

http://creativecommons.org/licenses/by/4.0/

(c) (i) Open Access

\begin{abstract}
A spectroscopic method for human sperm evaluation and characterization using Fourier Transform Infra Red (FTIR) is presented. The high sensitivity of FTIR to changes in chemical structure and arrangement of molecules and proteins makes it a powerful diagnostic tool. Our experimental results show that a simple MIR $\left(400 \mathrm{~cm}^{-1}-4000 \mathrm{~cm}^{-1}\right)$ transmission spectrum of a human sperm is very fast and can be used to determine the level of structure, compare to conventional LAB tests. No sample preparations are required, the semen has to be put on a special ZnSe substrate and inserted into the measurement compartment of the FTIR. Furthermore, this method can distinguish between immature sperm cell to white blood cell which by using a microscope is difficult and requires experience.
\end{abstract}

\section{Keywords}

Human Sperm, FTIR, Spectroscopic Measurements

\section{Introduction}

Diagnostics and analysis of human sperm are required as part of couples infertility investigation and treatments. It is also used to test human donors for sperm donation. The conventional tests done today are not perfect, time consuming and very expansive [1]. Furthermore, those tests are inaccurate since it depends on the experience of the lab technician [1].

Fourier Transform Infra-Red (FTIR) is used when measurements of optical properties of materials is required. Materials have unique molecular structures that cause them to absorb or transfer EM radiation as a function of radiation frequency [2]. This is due to molecular vibrations, rotations and electron spin flips. Table 1 shows the different types of absorption resonances. 
Table 1. Transition type according to MIR, FIR and MMW spectral bands [2].

\begin{tabular}{lcccc}
\hline \multicolumn{1}{c}{ Type of Radiation } & Frequency Range (Hz) & Wavelength Range & Wave Number $\left[\mathbf{c m}^{-1} \mathbf{]}\right.$ & Type of Transition \\
\hline Mid infrared & $10^{13}-10^{14}$ & $25 \mu \mathrm{m}-2.5 \mu \mathrm{m}$ & $400-4000$ & Molecular vibrations \\
Far infrared Millimeter waves & $3 \times 10^{11}-10^{13}$ & $1 \mathrm{~mm}-25 \mu \mathrm{m}$ & $10-400$ & $\begin{array}{l}\text { Molecular rotations, } \\
\text { electron spin flips }\end{array}$ \\
Microwaves Radio waves & $<3 \times 10^{11}$ & $>1 \mathrm{~mm}$ & $<10$ & Nuclear spin flips \\
\hline
\end{tabular}

The FTIR can measure transmission and refection in wide band of wave numbers $5 \mathrm{~cm}^{-1}-10,000 \mathrm{~cm}^{-1}$. Optical properties of solids, liquids, gasses and powders can be measured using the FTIR [2]. Since it has high sensitivity to changes in the chemical structure of the samples, it makes the spectral measurements attractive compare to X-ray diagnostics or other chemical conventional diagnostic methods. The principle of operation is based upon a Michelson interferometer with one displacement mirror and a fixed mirror. Sophisticated FFT procedure is used to obtain the signal as function of wave number [2].

Spectral measurements with Fourier Transform Infra Red (FTIR) are used to characterize the structure and composition of biomolecules [3]-[6]. Additional feasibility of this application is to diagnose different cancer types such as colonic cancer [7], lung cancer [8], malignant cancer fibroblast [9] and breast cancer [10]. FTIRs were used to investigate blood components in order to characterize infections disease in humans [11]. No sample preparation is required and the time required per sample is in the order of minutes and less. The diagnostic and analysis can be carried out for many samples in sequence.

In this publication we present our recent research in characterizing semen sample using FTIR spectroscopy in the Mid Infra Red (MIR) range. The components of the semen liquid include:

- Approximately 200 to 500 million spermatozoa_-(also called sperm or spermatozoans), produced in the testes, and released by ejaculation

- Amino acids, citrate, enzymes, flavins, fructose (the main energy source of sperm cells, which rely entirely on sugars from the seminal plasma for energy), phosphorylcholine, prostaglandins, (involved in suppressing an immune response by the female against the foreign semen), proteins, vitamin $\mathrm{C}$ - produced in the seminal vesicle

- Acid phosphatase, citric acid, fibrinolysin, prostate specific antigen, proteolytic enzymes, zinc (serves to help to stabilize the DNA-containing chromatin in the sperm cells. A zinc deficiency may result in lowered fertility because of increased sperm fragility. Zinc deficiency can also adversely affect spermatogenesis.) produced by the prostate.

- Galactose, mucus (serve to increase the motility of sperm cells in the vagina and cervix by creating a less viscous channel for the sperm cells to swim through, and preventing their diffusion out of the semen. Contributes to the cohesive jelly-like texture of semen.), pre-ejaculate, sialic acid—produced by bulbourethral gland.

All these components have influence on the semen function. Thus investigations of the optical properties of semen liquid can detect changes in the quantities and qualities of the samples. Preliminary measurements of optical properties of semen were made [12]. Those measurements show high absorption at $1650 \mathrm{~cm}^{-1}$ which corresponds to Amid 1 and $1545 \mathrm{~cm}^{-1}$ which corresponds to Amid 2 (see Figure 1).

In this research we characterize spectrally 80 semen sample using the FTIR. We also compared those optical results to standard semen analysis method [13] in the Male Infertility Center at Barzelai Medical Center, after receiving Helsinky approval.

\section{Experimental Results}

The semen samples were applied on a special ZnSe substrate. The ZnSe is transparent in the band $800 \mathrm{~cm}^{-1}$ $4000 \mathrm{~cm}^{-1}$ where the semen has absorption lines. The substrates with the semen were installed inside the sample compartment of the FTIR system. Figure 1 shows the absorption spectrum of normal human semen in the band $800 \mathrm{~cm}^{-1}-4000 \mathrm{~cm}^{-1}$. The absorption lines of Amid1 $\left(1650 \mathrm{~cm}^{-1}\right)$ and Amid2 $\left(1545 \mathrm{~cm}^{-1}\right)$ evolving from N-H, $\mathrm{C}=\mathrm{O}$ and $\mathrm{C}-\mathrm{H}$ connections are shown [14]. In Addition there are some weak absorption lines at $1245 \mathrm{~cm}^{-1}, 1450$ $\mathrm{cm}^{-1}, 1310 \mathrm{~cm}^{-1}$ and $1390 \mathrm{~cm}^{-1}$ evolving from $\alpha$-helical protein. More absorption lines are depicted in Figure 1.

In order to investigate the absorption lines of semen with different motilities we prepare three set of semen. 


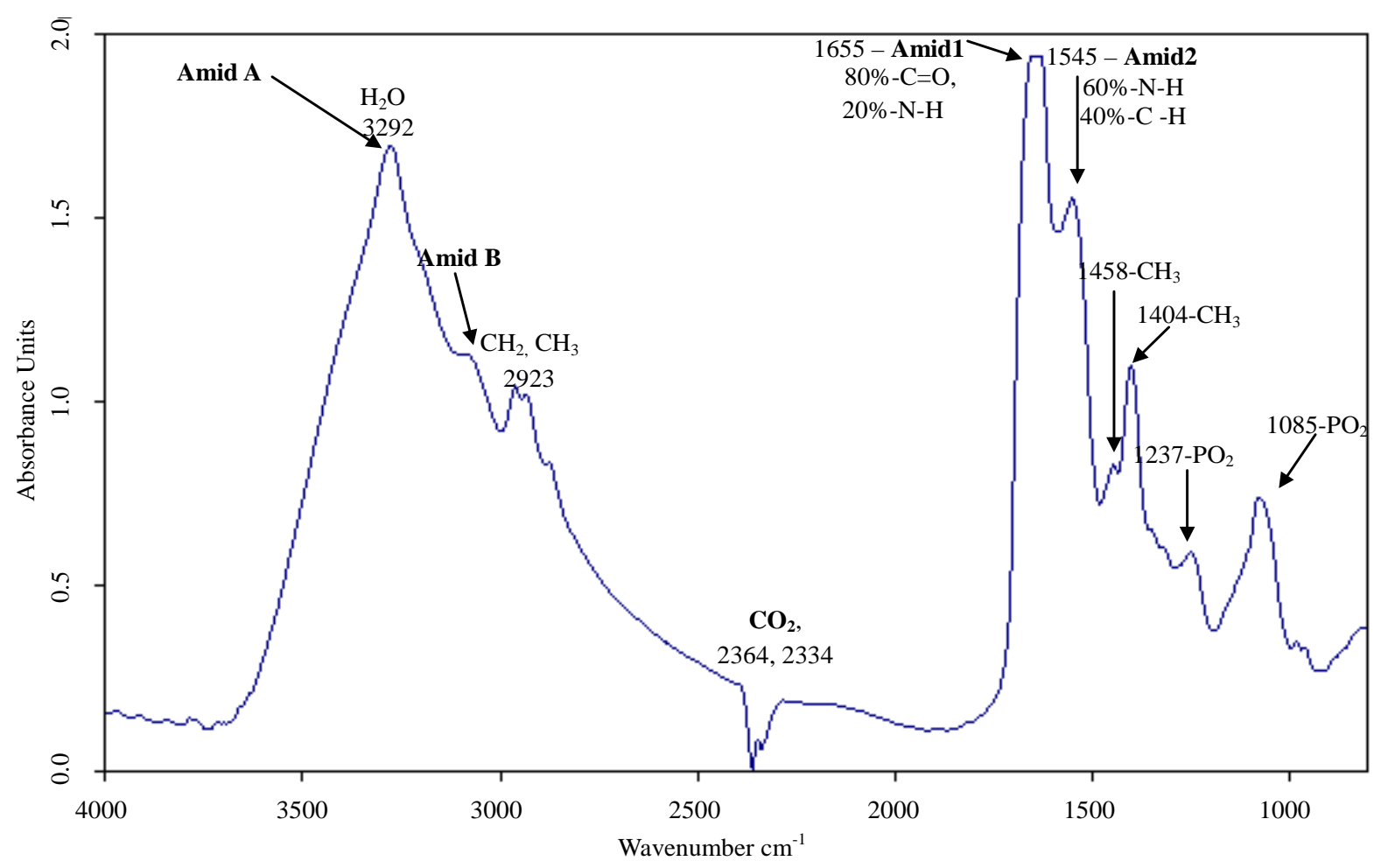

Figure 1. Absorption spectrum of normal human semen in the band $800-4000 \mathrm{~cm}^{-1}$.

Those semen were selected and grouped shortly after performing conventional diagnostic and analysis. The first set includes 8 normal semen motility samples the second includes 7 samples of $30 \%$ - 50\% semen motility and the third includes 65 sample of less than 30\% semen motility. The samples were put on the top of special ZnSe substrate and inserted into the FTIR measurement compartment. In this study, the spectral region between 800 $1800 \mathrm{~cm}^{-1}$ was investigated since it has many absorption lines as can be seen in Figure 1. Figure 2 shows the average absorption lines and the standard deviation of those three sets where the normal semen motility is in blue, the $30 \%-50 \%$ semen motility in green and lower than 30\% semen motility, in red.

The spectra of Figure 2 are with good agreement with the literature and with Figure 1. Note that the red line (less than 30\% motility) in less absorbing than the blue and green lines which indicates differences in the molecular concentration and structure.

\section{Discussion}

The FTIR was found to be an efficient diagnostic tool for the structure and the composition of biomolecules [2]. Previous researches with FTIR proved feasibility to characterize biological cell with high reliability [6]-[11], therefore we evaluated the spectroscopic characteristics in semen of fertile and infertile men. The semen (ejaculate) structure is derived from seminal plasma and cells, which determine the absorption spectrum by similarity to cells and blood plasma [10] [11]. Different compound of semen samples characterize in different sperm motility and concentration for example:

- Additional cells and different compound of seminal plasma can be in the semen sample due to prostate and sperm vesicle.

- Modification in the sperm cell volume in the testis, or obstacles between the testis to urethra cause to different sperm mobility and concentration in the ejaculate.

- Infection in the sperm blister and prostate cause to different in the seminal plasma and sperm cells.

The absorption spectrum of seminal plasma and blood plasma are different because of the volume of proteins, hormones and electrolytes. The sperm cell motility in the ejaculate categorizes the group of the samples (Fertile and Infertile). Execute baseline correction and normalization according to Amid1 spectrum absorption allow to revaluate the quality and the quantity of the semen sample. There is no direct correlation between specific wave 


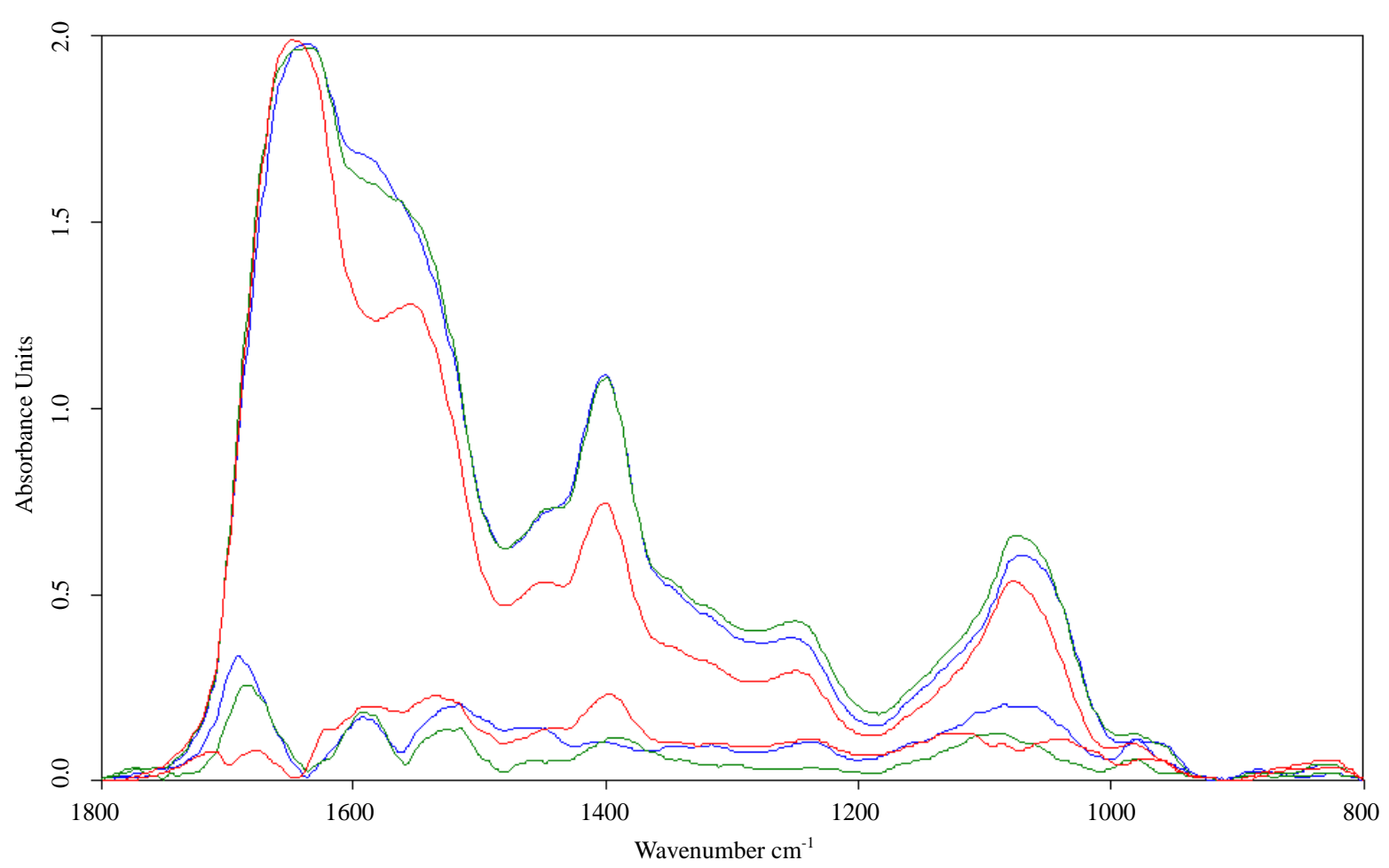

Figure 2. Absorption spectrum and standard deviation of three sets of different semen motility 1) normal blue line 2) $30 \%$ $50 \%$ green line and 3 ) less than $30 \%$ red line.

numbers absorption to motility parameter, however the spectrum absorption is continuous $1700-800 \mathrm{~cm}^{-1}$. The absorption spectrum can receive from chemical reaction that found in the cells and in the seminal plasma, changes in the seminal plasma or in the cells can cause to identify absorption spectrum although that the source of the change is different. In the future it is important to measure the absorption spectrum of all the semen components individually in order to compare the absorption spectrum of specific component that contain proteins, amino acids and chemical connection to references absorption spectrums, the purpose is to notice if we can get more remarkable different curves in the absorption spectrum. These curves enable us more accuracy identification of substance in the seminal plasma and in sperm cells. The spectroscopy can give additional information on sperm quality after treatment of infertile men and can guide to better methods of characterizing and treating patients. The characterization of DNA, important structure of nucleus and sperm tail can help to select a single sperm with better potential for injection in vitro fertilization. This method can help to determine changes in the sperm cell as results from freezing and thawing before surgical treatment, or in vitro fertilization. Based on the capability of the method to identify specific chemical and molecular connections, we can assist to characterize the different compositions of cells inside the testis biopsy in order to understand the testis cells characteristics that are a part of the spermatozoa process, for improving the quantity and quality in sperm cells production.

\section{Acknowledgements}

The authors would like to thanks Dr. S. Segal from Barzilai Medical Center, Male Infertility Center, for the sperm samples.

\section{References}

[1] Esteves, S.C., Miyaoka, R. and Agarwal, A. (2011) An Update on the Clinical Assessment of the Infertile Male. Clinics (Sao Paulo), 66, 691-700. http://dx.doi.org/10.1590/S1807-59322011000400026

[2] Griffiths, P. and de Haseth, J. (1986) Fourier Transform Infrared Spectrometry. 2nd Edition, Wiley \& Sons, Hoboken, NJ, 1-18.

[3] Wood, B., Quinn, M., Tait, B., Ashdown, M., Hislop, T., Romeo, M. and Mcnaughton, D. (1997) FTIR Micro Spec- 
troscopic Study of Cell Types and Potential Confounding Variables in Screening for Cervical Malignancies. Biospectroscopy, 4, 75-91. http://dx.doi.org/10.1002/(SICI)1520-6343(1998)4:2<75::AID-BSPY1>3.0.CO;2-R

[4] Priyangika Bandaranayake, K.M., Sivakumar, V., Wang, R. and Hastings, G. (2006) Modeling the $A_{1}$ Binding Site in Photosystem I. Density Functional Theory for the Calculation of “Anion-Neutral” FTIR Difference Spectra of Phylloquinone. Vibrational Spectroscopy, 42, 78-87. http://dx.doi.org/10.1016/j.vibspec.2006.01.003

[5] Schweitzer-Stenner, R. (2006) Advances in Vibrational Spectroscopy as a Sensitive Probe of Peptide and Protein Structure: A Critical Review. Vibrational Spectroscopy, 42, 98-117. http://dx.doi.org/10.1016/j.vibspec.2006.01.004

[6] Michael, S., Sevegney, A., Rangaramanujam, M., Kannan, A., Allen, R., Siedle, B., Ratna Naik, C., Vaman, M. and Naik, D. (2006) Vibrational Spectroscopic Investigation of Stereoregularity Effects on Syndiotactic Polypropylene Structure and Morphology. Vibrational Spectroscopy, 40, 246-256. http://dx.doi.org/10.1016/j.vibspec.2005.10.003

[7] Ramesh, J., Salman, A., Mordechai, S., Argov, S., Goldstein, J. and Sinelnikov, I. (2001) FTIR Microscopic Studies on Normal, Polyp, and Malignant Human Colonic Tissues. Subsurface Sensing Technologies and Application, 2, 99-117. http://dx.doi.org/10.1023/A:1011570719170

[8] Wang, H.P., Wang, H.C. and Huang, Y.J. (1997) Microscopic FTIR Studies of Lung Cancer Cell in Pleural Fluid. Science of the Total Environment, 204, 283-287. http://dx.doi.org/10.1016/S0048-9697(97)00180-0

[9] Salman, A., Argov, S., Ramesh, J., Ziad, H., Goldstein, J., Sinelnikov, I., Guterman, H. and Mordechai, S. (2001) FT-IR Microscopic Characterization of Normal and Malignant of Human Colonic Tissues. Cellular and Molecular Biology, 22, 159-166.

[10] Dukor, R.K., Liebman, M.N. and Johnson, B. (1998) A New Non-Destructive Method for Analysis of Clinical Samples with FTIR-Microspectroscopy. Breast Cancer as an Example. Cellular \& Molecular Biology, 44, 211-217.

[11] Mordehai, J., Ramesh, J., Huleihel, M., Cohen, Z., Kleiner, O., Talyshinnsky, M., Errukhimovitch, V., Kahana A. and Mordechai, S. (2002) FTIR Microscopic Characterization of Blood Components from Patients with Infectious Diseases. Journal of Biomedical Optics, 2, 66-78.

[12] Wood, B., Quinn, M., Tait, B., Ashdown, M., Hislop, T., Romeo, M. and Mcnaughton, D. (1997) FTIR Micro Spectroscopic Study of Cell Types and Potential Confounding Variables in Screening for Cervical Malignancies. Biospectroscopy, 4, 75-91. http://dx.doi.org/10.1002/(SICI)1520-6343(1998)4:2<75::AID-BSPY1>3.0.CO;2-R

[13] Carver-Wa, J.A. and Hollanders, J.M.G. (1996) Male Infertility from A to Z: A Concise Encyclopedia. CRC Press, Boca Raton, 1-198.

[14] Mantsch, H.H. and Champmann, D. (1996) Infrared Spectroscopy of Biomolecules. Wiley-Liss, New York, 1-359. 